\title{
Cytokine Imbalance as a Common Mechanism in Both Psoriasis and Rheumatoid Arthritis
}

\author{
Yong Tan, ${ }^{1}$ Qiu Qi, ${ }^{2}$ Cheng Lu, ${ }^{1}$ Xuyan Niu, ${ }^{1}$ Yanping Bai, ${ }^{3}$ Chunyan Jiang, \\ Yang Wang, ${ }^{5}$ Youwen Zhou, ${ }^{6,7}$ Aiping Lu, ${ }^{1,8,9}$ and Cheng Xiao ${ }^{10}$ \\ ${ }^{1}$ Institute of Basic Research in Clinical Medicine, China Academy of Chinese Medical Sciences, Beijing 100700, China \\ ${ }^{2}$ Institute of Clinical Pharmacology, Beijing An Zhen Hospital, Capital Medical University, Beijing 100029, China \\ ${ }^{3}$ Department of Dermatology, China-Japan Friendship Hospital, Beijing 100029, China \\ ${ }^{4}$ Department of Dermatology, Beijing Hospital of Traditional Chinese Medicine, Beijing 100010, China \\ ${ }^{5}$ Department of Dermatology and Venerology, Peking University First Hospital, Beijing 100034, China \\ ${ }^{6}$ Department of Dermatology and Skin Science, University of British Columbia, Vancouver, BC, Canada \\ ${ }^{7}$ Molecular Medicine Laboratory and Chieng Genomics Center, Vancouver Coastal Health Research Institute, Vancouver, BC, Canada \\ ${ }^{8}$ Institute for Advancing Translational Medicine in Bone \& Joint Diseases, School of Chinese Medicine, Hong Kong Baptist University, \\ Kowloon Tong, Hong Kong \\ ${ }^{9}$ E-Institute of Chinese Traditional Internal Medicine, Shanghai Municipal Education Commission, Shanghai 201203, China \\ ${ }^{10}$ Institute of Clinical Medicine, China-Japan Friendship Hospital, Beijing 100029, China
}

Correspondence should be addressed to Youwen Zhou; youwen.zhou@ubc.ca, Aiping Lu; aipinglu@hkbu.edu.hk, and Cheng Xiao; xc2002812@126.com

Received 11 October 2016; Accepted 30 November 2016; Published 25 January 2017

Academic Editor: Qingdong Guan

Copyright (C) 2017 Yong Tan et al. This is an open access article distributed under the Creative Commons Attribution License, which permits unrestricted use, distribution, and reproduction in any medium, provided the original work is properly cited.

Psoriasis (PS) and rheumatoid arthritis (RA) are immune-mediated inflammatory diseases. Previous studies showed that these two diseases had a common pathogenesis, but the precise molecular mechanism remains unclear. In this study, RNA sequencing of peripheral blood mononuclear cells was employed to explore both the differentially expressed genes (DEGs) of 10 PS and 10 RA patients compared with those of 10 healthy volunteers and the shared DEGs between these two diseases. Bioinformatics network analysis was used to reveal the connections among the shared DEGs and the corresponding molecular mechanism. In total, 120 and 212 DEGs were identified in PS and RA, respectively, and 31 shared DEGs were identified. Bioinformatics analysis indicated that the cytokine imbalance relevant to key molecules (such as extracellular signal-regulated kinase 1/2 (ERK1/2), p38 mitogen-activated protein kinase (MAPK), tumor necrosis factor (TNF), colony-stimulating factor 3 (CSF3), interleukin- (IL-) 6, and interferon gene (IFNG)) and canonical signaling pathways (such as the complement system, antigen presentation, macropinocytosis signaling, nuclear factor-kappa B (NF- $\kappa$ B) signaling, and IL-17 signaling) was responsible for the common comprehensive mechanism of PS and RA. Our findings provide a better understanding of the pathogenesis of PS and RA, suggesting potential strategies for treating and preventing both diseases. This study may also provide a new paradigm for illuminating the common pathogenesis of different diseases.

\section{Introduction}

Psoriasis (PS) and rheumatoid arthritis (RA) are immunemediated chronic inflammatory diseases. PS is characterized by epidermal hyperplasia, and the predominant pathological feature of RA is the destruction of synovial joints. Studies have increasingly suggested that patients suffering from PS or RA have similarly increased risks of certain disorders, such as major adverse cardiovascular events, malignancy, and liver fatty changes, compared to the general population [1-6]. Additionally, these two diseases have a similar pathogenesis. Previous studies have indicated that the chronic inflammation mediated by T helper (Th) 17 and Thl cells plays a key role in PS $[7,8]$. Cytokines, including Thl-related (tumor necrosis factor- (TNF-) $\alpha$, interferon gamma (IFN $\gamma$ ), and interleukin- (IL-) 2) and Th17-related (IL-17A, IL-17F, IL-22, 
IL-26, and TNF- $\alpha$ ) proteins, together with IL-23, IL-20, and IL-15 were increased in the sera of PS patients $[8,9]$. For $\mathrm{RA}$, the concerted interaction of proinflammatory cytokines also plays a dominate role in its pathogenesis [10]. An increasing number of clinical and histopathological features of PS and RA are explained by an imbalance in particular cytokines, which is one of the most fascinating research topics inspiring researchers [9]. The similarities between the mechanisms involved in PS and RA imply an underlying genetic homogeneity of these two diseases. By exploring the genetic overlap of PS and RA, we seek to provide a better understanding of their molecular correlation and their shared mechanisms. The genetic commonality of PS and RA may provide increasing evidence for developing combined treatment targets for both diseases. These targets will eventually complement the traditional systemic treatments and biological agents that are currently available.

Due to the progress in high-throughput techniques for biological research, next-generation sequencing (NGS) platforms are often used to explore the gene profile; these platforms have the advantages of greater sensitivity and more precise quantification, thus providing a more complete picture of the transcriptome in studies of gene expression than that obtained by microarrays [11]. Measurements of mRNA expression by RNA sequencing are valuable for identifying the molecular changes that occur in cells, thus providing clues regarding the molecular networks involved in disease processes [12]. Studies have focused on molecular changes in PS or RA independently using transcriptome or gene expression profile technology $[12,13]$, but few reports have been published concerning the correlations between PS and RA at the transcriptome level, including in-depth studies of the mechanisms and molecular networks involved in the pathogenesis common to RA and PS.

The present study applied RNA sequencing technology to the peripheral blood mononuclear cell (PBMC) RNA of PS and RA patients and healthy volunteers, and differentially expressed genes (DEGs) were explored among the groups. Furthermore, bioinformatics analysis was performed to identify the key molecules and signaling pathways relevant to RA and PS as well as the upstream regulators related to the identified genes. This study aimed to obtain a comprehensive understanding of the cytokine imbalance in RA and PS based on DEGs, which may provide new insights into the pathogenesis of and suitable prevention strategies for these two diseases.

\section{Materials and Methods}

2.1. Patients. PS patients, RA patients, and healthy volunteers were recruited from the China-Japan Friendship Hospital in Beijing City of China at the dermatology clinic, the rheumatology clinic, and the health screening center, respectively. The diagnosis of PS was consistent with the guidelines of the care for the management of psoriasis from the American Academy of Dermatology and the guidelines for the treatment of psoriasis from the Psoriasis Study Group of Chinese Medical Association [14, 15]. Additionally, enrolled patients had no symptoms or signs of psoriatic arthritis. These patients had a psoriasis area severe index (PASI) greater than 10 or body surface area (BSA) greater than $10 \%$ but a PASI less than 30 and BSA less than $30 \%$. A diagnosis of RA was based on the 1987 American College of Rheumatology revised criteria and the 2010 American College of Rheumatology/European League against Rheumatism classification criteria for RA. Disease activity was assessed by the Disease Activity Score in 28 joints (DAS28). For inclusion, the control subjects could not have a history of an arthritic disorder and were subject to the same exclusion criteria as the PS and RA patients. Given that RA is two- to threefold more common in females than males, only females were chosen as the observed subjects in this study [16].

The following subjects were excluded: individuals who were $\geq 65$ years old and $\leq 18$ years old; individuals with complications, such as cardiovascular and cerebrovascular diseases, respiratory, digestive, urinary, and hematological diseases, metabolic syndrome, and mental disturbances; individuals who were pregnant, lactating, or who planned to become pregnant within a year; individuals who received topical treatments (such as corticosteroids or retinoic acid) within 2 weeks, systemic therapy within 4 weeks, or biological therapy within 12 weeks; PS patients with a concurrent RA diagnosis; and RA patients diagnosed with any type of PS.

In summary, 10 female PS patients, 10 female RA patients, and 10 female healthy controls were enrolled into this study. All protocols involving human subjects were approved by the ethics committee of the China-Japan Friendship Hospital (ethics ID: 2014-58), and informed consent was signed by all participants before the study began.

2.2. PBMC Isolation and Total RNA Extraction. In all, $3 \mathrm{~mL}$ peripheral fasting blood samples were collected from all subjects in the morning. PBMCs were isolated using density gradient centrifugation. Specifically, based on FicollHypaque gradient solution (Histopaque-1077, Sigma-Aldrich, USA), $3 \mathrm{~mL}$ of heparinized whole blood was diluted to $6 \mathrm{~mL}$ with phosphate-buffered saline (PBS, $\mathrm{pH} 7.4$ ), layered on top of $3 \mathrm{~mL}$ of Histopaque and centrifuged for $30 \mathrm{~min}$ at $400 \times$ g. PBMCs were aspirated, washed twice, suspended in PBS, and counted with a hemocytometer. PBMCs were lysed in Trizol reagent $\left(1 \mathrm{~mL} / 1 \times 10^{7}\right.$ PBMCs) (Invitrogen, Karlsruhe, Germany; Carlsbad, CA) and stored at $-80^{\circ} \mathrm{C}$ for the subsequent testing. Total RNA in PBMC sample was isolated using the Trizol extraction method, and it was quantified with a NanoDrop ND-1000 spectrophotometer (Thermo Fisher Scientific Inc., Marietta, OH, USA). The RNA Integrity Number was greater than 7.0, and acceptable quality values accorded with A260/A280 ratios ranging from 1.8 to 2.2 for each total RNA sample.

2.3. Identification of DEGs. Total RNA of each sample was purified by adsorption of biotin oligo magnetic beads. cDNA synthesis was conducted after the binding of mRNA. Doublestranded cDNA was introduced to the cDNA fragment digested by NlaIII endonuclease, and the bound fragments contained CATG sites and adjacent poly A tails at the $3^{\prime}$ end. After precipitation of the $3^{\prime}$ cDNA fragment, Illumina adaptor 1 was added to the $5^{\prime}$ end. Both the adaptor 1 
and CATG sites are recognized by MmeI, which cuts at a downstream CATG site and produces fragments of 17-bp tags with adaptor 1 . Adaptor 2 was added to the $3^{\prime}$ end of these tags after the fragment was removed using beads attached to the $3^{\prime}$ end. Then, these sequences were prepared for Solexa sequencing [17].

Clean tags were produced by filtering the adaptor sequences and removing low-quality sequences (containing ambiguous bases). Only the tags with perfect matches or one mismatch were further considered and annotated based on the reference genes. The expression level of each gene was estimated by the frequency of clean tags and then normalized to TPM (number of transcripts per million clean tags), which is a standard method extensively used in DEG analysis [18]. The number of tags mapped to a given gene represented the expression level of this gene. Expression levels of a gene from two different samples were compared to provide an expression difference. Significance values for differences in expression were determined using a modified exact test. The gene was classified as differentially expressed only when the expression difference was greater than 1.2 -fold with a $p$ value less than 0.01 .

2.4. Bioinformatics Analysis about DEGs. The information of shared DEGs identified in PS and RA was uploaded into the Ingenuity Pathways Analysis system (IPA, Ingenuity Systems, http://www.ingenuity.com). The "Core Analysis" module in IPA was utilized to analyze and visualize interactions of the shared DEGs. These interactions were characterized by specific canonical pathways and molecular networks. Analytical score was the negative base 10 logarithm of Fisher's exact test $p$ value in canonical pathway analysis. Significance for biological functions of each network was symbolized by a $p$ value for the enrichment of the genes in the network by comparison with the entire Ingenuity Pathway Knowledge Base.

\section{Results}

3.1. Baseline Characteristics of Study Subjects. The characteristics of the enrolled subjects, including age, disease duration, BMI, PASI, BSA, and results of blood routine and biochemical tests, are presented in Table 1. No significant differences in any of the examination indicators were noted among the groups.

3.2. Identified Shared DEGs between PS and RA and the Corresponding Functions. One hundred and twenty genes in PS and 212 genes in RA were identified as DEGs when compared with the controls (Figure 1, Tables S1 and S2 in Supplementary Material available online at https://doi.org/ 10.1155/2017/2405291). As shown in Figure 1 and Table 2, there were 31 shared genes between PS and RA, including 20 upregulated and 11 downregulated DEGs, which reflects the complex association of PS and RA at the transcriptome level. The biological functions corresponding to the shared DEGs mainly include cell-to-cell signaling, systemic autoimmune syndrome, cell death and apoptosis, inflammatory dermatoses, and rheumatic arthritis (Figure 2, Table S3).
TABLE 1: Characteristics of the enrolled subjects for the three groups.

\begin{tabular}{lccc}
\hline Characteristic & Control $(n=10)$ & PS $(n=10)$ & RA $(n=10)$ \\
\hline $\begin{array}{l}\text { Age (years) } \\
\begin{array}{l}\text { Disease duration } \\
\text { (years) }\end{array}\end{array}$ & $45.80 \pm 3.50$ & $48.60 \pm 5.20$ & $54.50 \pm 7.10$ \\
BMI $\left(\mathrm{kg} / \mathrm{m}^{2}\right)$ & $22.34 \pm 1.83$ & $23.23 \pm 4.71$ & $25.81 \pm 2.62$ \\
PASI & $/$ & $9.17 \pm 8.56$ & $/$ \\
BSA $(\%)$ & $/$ & $19.40 \pm 4.14$ & $/$ \\
ESR $(\mathrm{mm} / \mathrm{h})$ & $/$ & $/$ & $39.14 \pm 29.53$ \\
CRP $(\mathrm{mg} / \mathrm{L})$ & $/$ & $/$ & $15.48 \pm 15.40$ \\
RF $(\mathrm{IU} / \mathrm{mL})$ & $/$ & $/$ & $63.76 \pm 71.81$ \\
$\mathrm{WBC}\left(\times 10^{9} / \mathrm{L}\right)$ & $4.92 \pm 1.10$ & $5.33 \pm 0.98$ & $6.09 \pm 1.50$ \\
$\mathrm{HGB}(\mathrm{g} / \mathrm{L})$ & $126.20 \pm 13.89$ & $124.72 \pm 11.54$ & $122.38 \pm 14.74$ \\
PLT $\left(\times 10^{9} / \mathrm{L}\right)$ & $228.70 \pm 28.69$ & $251.19 \pm 34.56$ & $246.75 \pm 75.06$ \\
\hline
\end{tabular}

Note. Comparisons of clinical indicators of the PS group, RA group, and control group. An unpaired $t$-test was used for continuous variables analysis, and the data are expressed as the mean \pm SD when appropriate $(95 \% \mathrm{CI})$.

3.3. Networks of the Shared DEGs and the Corresponding Functions. To reveal the connections between the shared DEGs, the biomolecular networks of these DEGs were constructed using IPA. As shown in Figure 3, these DEGs were associated with one another directly or indirectly, and three networks were established. Highly linked molecules of the networks included extracellular signal-regulated kinase 1/2 (ERK1/2), p38 mitogen-activated protein kinase (MAPK), interferon gene (IFNG), and $\mathrm{Ca}^{2+}$. The network functions included organismal injury and abnormalities, cell death and survival, and cellular function and maintenance.

3.4. Signaling Pathways Relevant to the Merged Bionetwork. The three networks were merged and formed a large network that was associated with 42 signaling pathways. TNF was highly linked molecule of the network. The main categories corresponding to these signaling pathways were cytokine signaling, cellular immune response, and humoral immune pathways. Signaling pathways with $-\log (p$-value) more than $2.00 E+00$ represented the most significantly relevant pathways related to the merged network and included the complement system, antigen presentation, macropinocytosis signaling, acute phase response signaling, nuclear factorkappa B (NF- $\kappa$ B) signaling, IL-6 signaling, IL-17 signaling, and p38 MAPK signaling (Figures 4 and 5 and Table S4). Top five pathways of these pathways were associated with the shared DEGs.

3.5. Upstream Regulators of the Shared DEGs. Thirty-six upstream regulators were identified by biomolecular network analysis, with the majority being cytokine molecules, that is, $52.78 \%$ of them (Figure 6(a), Table S5). The regulators with $p$ values less than $1.00 E-04$ included colony-stimulating factor 3 (CSF3), IL-6, FOS, p38 MAPK, and TNF, and the connection between every regulator and the corresponding target molecule is presented in Figure 6(b). The main biofunctions of the regulated effect networks corresponding 
PS

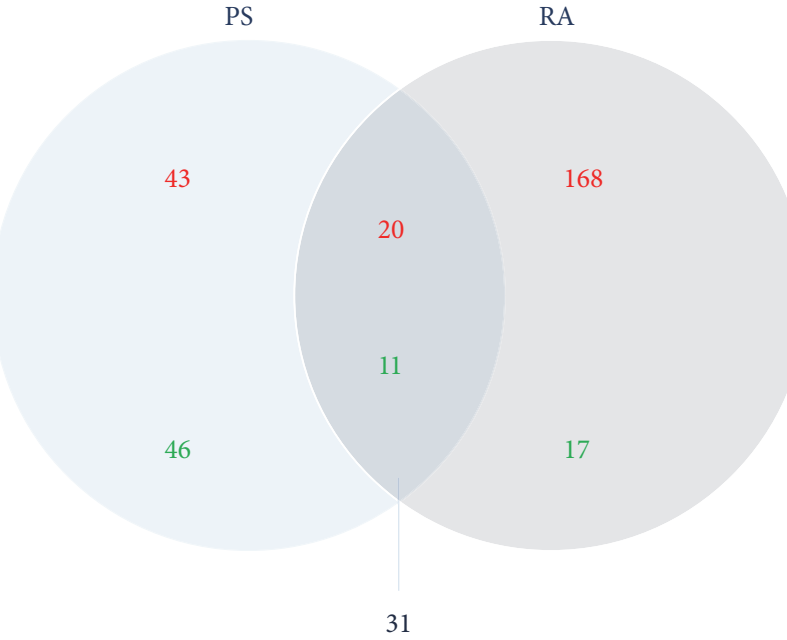

Shared DEGs
Upregulated

Downregulated

(a)

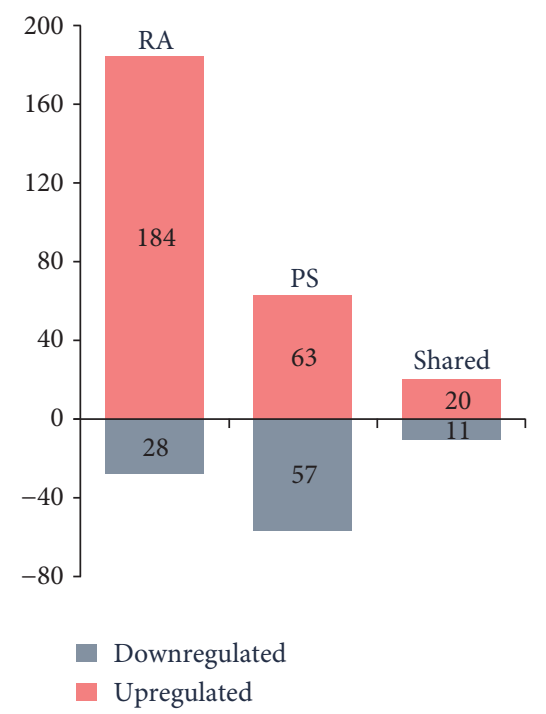

(b)

Figure 1: Number of DEGs in PS and RA. (a) The Venn diagram indicates the number of uniquely upregulated (red) or downregulated (green) genes from the comparisons of PS and RA with control and the number of shared DEGs. (b) The bar diagram shows the number of DEGs in PS, RA, and the shared DEGs between them.

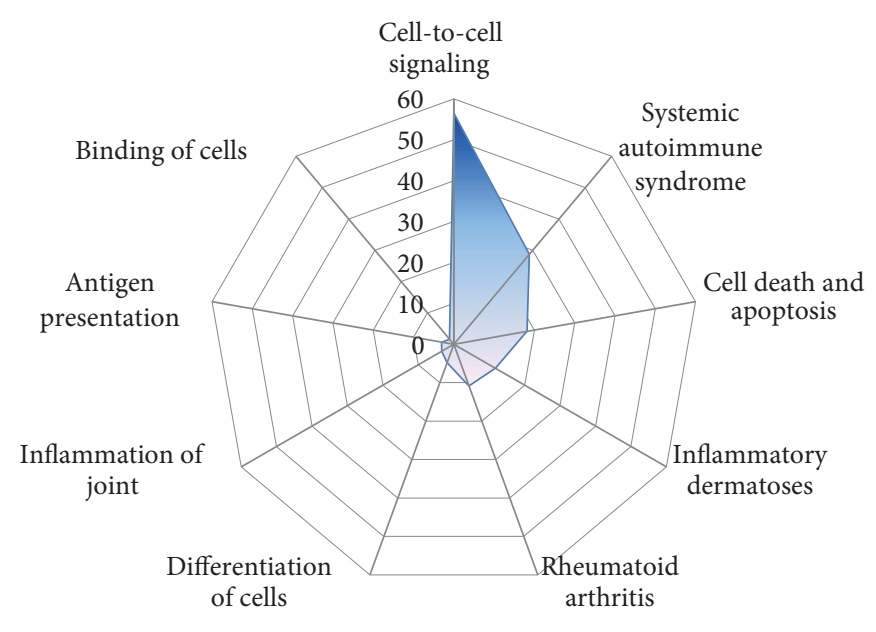

Significance

FIGURE 2: Biological functions corresponding to the shared DEGs.

to those regulators were inflammation or immune-related processes.

\section{Discussion}

PS and RA are immune-mediated inflammatory diseases. An increasing number of studies have reported a correlation between PS and RA, but the exact common molecular mechanisms have not been elucidated. With the development of high-throughput detection and analysis techniques, including genomics and bioinformatics, the exploration of these comprehensive mechanisms has become feasible. PBMCs can be extracted from whole blood and consist of lymphocytes ( $\mathrm{T}$ cells, B cells, and NK cells) and monocytes. Identifying gene expression in PBMCs is an important strategy to determine disease-specific genes in holism [19]. Methodologically, by comparison of gene profiles of patients with particular disease and healthy persons, the disease-specific genes can be found [20]. In this study, the PBMC gene profiles of PS patients, RA patients, and healthy 
TABLE 2: The shared DEGs in PS and RA.

\begin{tabular}{|c|c|c|c|c|}
\hline \multirow{2}{*}{$n$} & \multirow{2}{*}{ Symbol } & \multirow{2}{*}{ Entrez gene name } & \multicolumn{2}{|c|}{ Fold change } \\
\hline & & & PS & RA \\
\hline 1 & AEBP1 & AE binding protein 1 & 2.615 & 2.644 \\
\hline 2 & ARG1 & Arginase 1 & 1.295 & 2.224 \\
\hline 3 & C1QB & Complement component 1 , q subcomponent, B chain & 1.153 & 2.356 \\
\hline 4 & C1QC & Complement component 1 , q subcomponent, $\mathrm{C}$ chain & 1.177 & 3.000 \\
\hline 5 & C4BPA & Complement component 4 binding protein alpha & 1.504 & 3.322 \\
\hline 6 & CACNG6 & Calcium voltage-gated channel auxiliary subunit gamma 6 & -1.329 & -1.445 \\
\hline 7 & CCNB2 & Cyclin B2 & 1.479 & 2.390 \\
\hline 8 & CD177 & CD177 molecule & 1.000 & 2.404 \\
\hline 9 & CEP55 & Centrosomal protein 55 & 1.303 & 1.554 \\
\hline 10 & CHI3L1 & Chitinase 3 like 1 & 1.095 & 1.258 \\
\hline 11 & CTGF & Connective tissue growth factor & 4.700 & 4.954 \\
\hline 12 & DAAM1 & Dishevelled associated activator of morphogenesis 1 & -1.158 & -1.489 \\
\hline 13 & EGR2 & Early growth response 2 & 1.413 & 2.495 \\
\hline 14 & FHDC1 & FH2 domain containing 1 & 1.122 & 1.631 \\
\hline 15 & FOLR3 & Folate receptor 3 (gamma) & 1.787 & 1.853 \\
\hline 16 & HLA-DQA2 & Major histocompatibility complex, class II, DQ alpha 2 & 1.548 & 1.305 \\
\hline 17 & HLA-DRB4 & Major histocompatibility complex, class II, DR beta 4 & 1.884 & 1.711 \\
\hline 18 & IFI27 & Interferon alpha inducible protein 27 & 1.948 & 2.764 \\
\hline 19 & ITGB4 & Integrin subunit beta 4 & -1.531 & -2.478 \\
\hline 20 & KRT1 & Keratin 1 & -1.142 & -1.415 \\
\hline 21 & LTF & Lactotransferrin & 1.526 & 1.708 \\
\hline 22 & MET & MET protooncogene, receptor tyrosine kinase & 4.700 & 4.459 \\
\hline 23 & MT2A & Metallothionein $2 \mathrm{~A}$ & -1.705 & -1.253 \\
\hline 24 & PGLYRP1 & Peptidoglycan recognition protein 1 & 1.535 & 1.907 \\
\hline 25 & RNF182 & Ring finger protein 182 & 3.138 & 3.459 \\
\hline 26 & SLC26A8 & Solute carrier family 26 member 8 & 1.890 & 3.021 \\
\hline 27 & SNAI1 & Snail family zinc finger 1 & -2.149 & -1.672 \\
\hline 28 & TECPR1 & Tectonic beta-propeller repeat containing 1 & -1.027 & -1.240 \\
\hline 29 & THEM5 & Thioesterase superfamily member 5 & -1.476 & -1.306 \\
\hline 30 & TNFSF11 & Tumor necrosis factor superfamily member 11 & -2.000 & -3.000 \\
\hline 31 & YEATS2 & YEATS domain containing 2 & -1.631 & -1.304 \\
\hline
\end{tabular}

volunteers were evaluated by determining the DEGs, and 31 gene expression signatures commonly shared between PS and RA were identified. Based on these shared DEGs, the pathogenesis common to both PS and RA was elucidated at the transcriptome level. The discoveries of this study suggest that the common mechanism of PS and RA mainly involves inflammation and an abnormal immune response characterized by a cytokine imbalance. Specifically, the identified highly linked molecules, significant signaling pathways, and upstream regulators were directly or indirectly associated with the regulation of a variety of cytokines. As shown in Figure 7, certain key molecules (ERK1/2, CSF3, FOS, IFNG, and TNF) and significant signaling pathways (the complement system, antigen presentation, macropinocytosis signaling, acute phase response signaling, NF- $\kappa \mathrm{B}$ signaling, IL-6 signaling, IL-17 signaling, and p38 MAPK signaling) were associated with an imbalance of cytokines; this imbalance may provide new clues for a better understanding of PS and RA.
The complement system is an essential component of innate immunity, and it plays an important role in modulating adaptive immunity. Its activation contributes to the pathogenesis of autoimmune and inflammatory diseases, such as PS and RA [21]. Reduction of complement activation is one of the mechanisms by which TNF- $\alpha$ inhibitors exert their effectiveness in these two diseases [22]. In this study, the upregulation of three DEGs (C4BPA, C1QB, and C4BPA) implied that the complement system was activated, which was consistent with previous studies. This study further confirmed that the complement system is indeed an attractive therapeutic target for both PS and RA. Professional antigenpresenting cells, such as dendritic cells (DCs), macrophages, and $\mathrm{B}$ cells, play a key role in triggering and/or maintaining the chronic inflammatory process in RA [23]. Increasing evidence indicates that RA treatment may occur through the manipulation of antigen presentation [24]. This study found that the activated antigen presentation pathway is characterized by upregulation of two DEGs, HLA-DQA2 and 

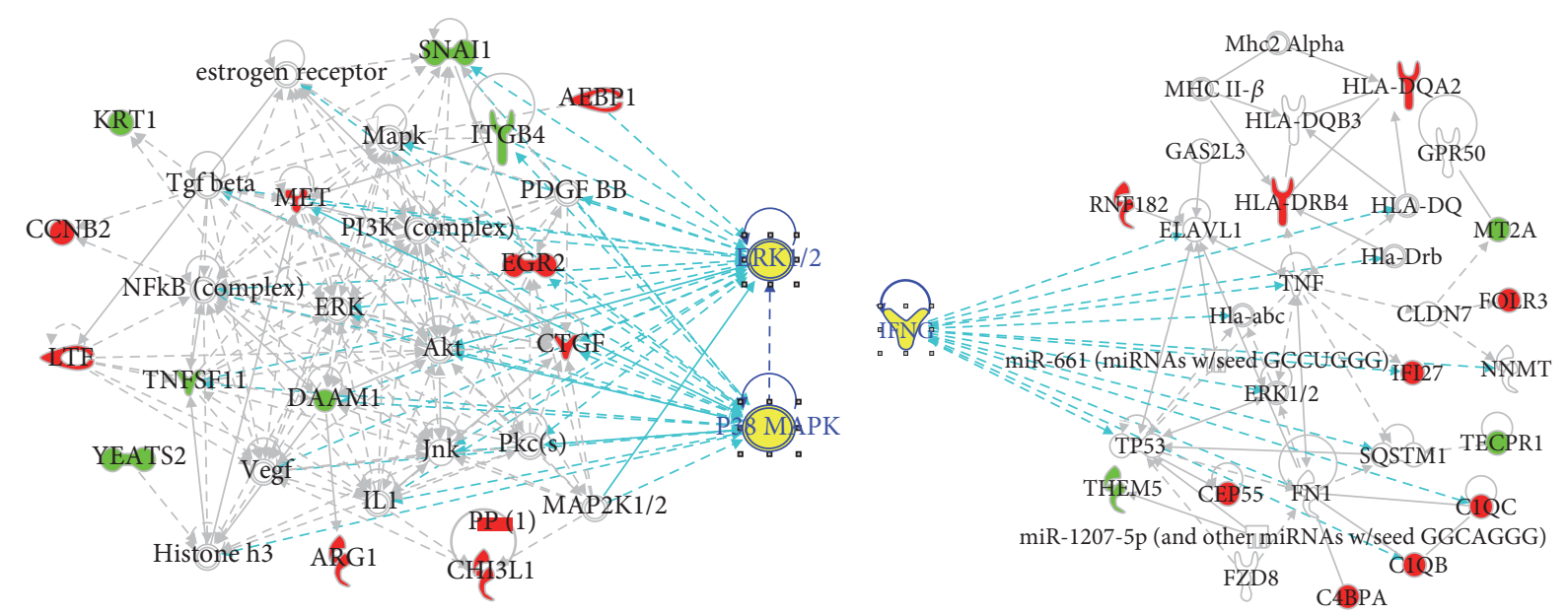

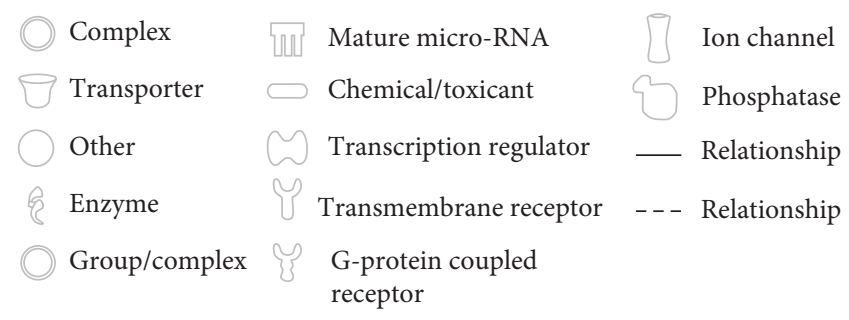

(a)

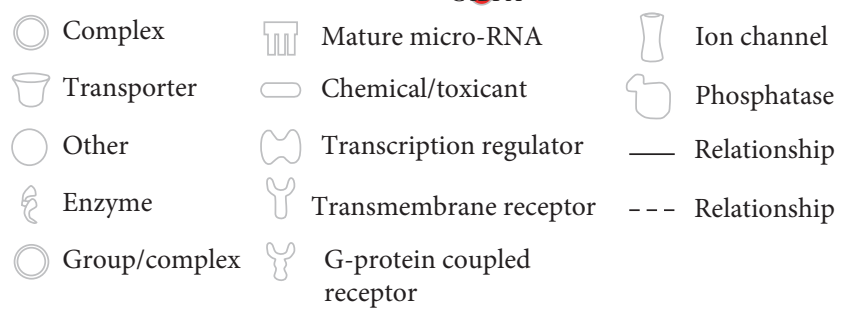

(b)

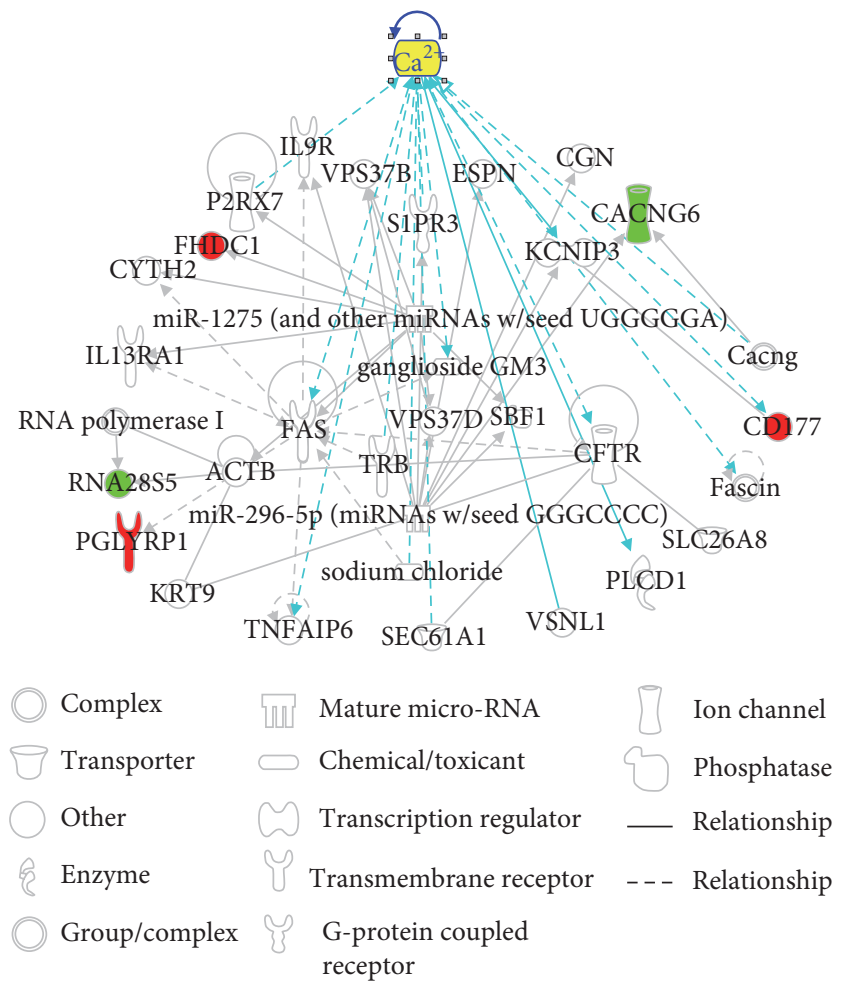

(c)

FIGURE 3: Biomolecular networks related to the shared DEGs. In each network, molecules are represented as nodes, and the biological relationship between two nodes is represented as a line. Red symbols represent upregulated DEGs; green symbols represent downregulated DEGs. Yellow symbols indicate the highly linked molecules from the Ingenuity Knowledge Database. Solid lines between molecules indicate a direct physical relationship between molecules, whereas dash lines represent indirect functional relationships. (a) The first network. (b) The second network. (c) The third network. 


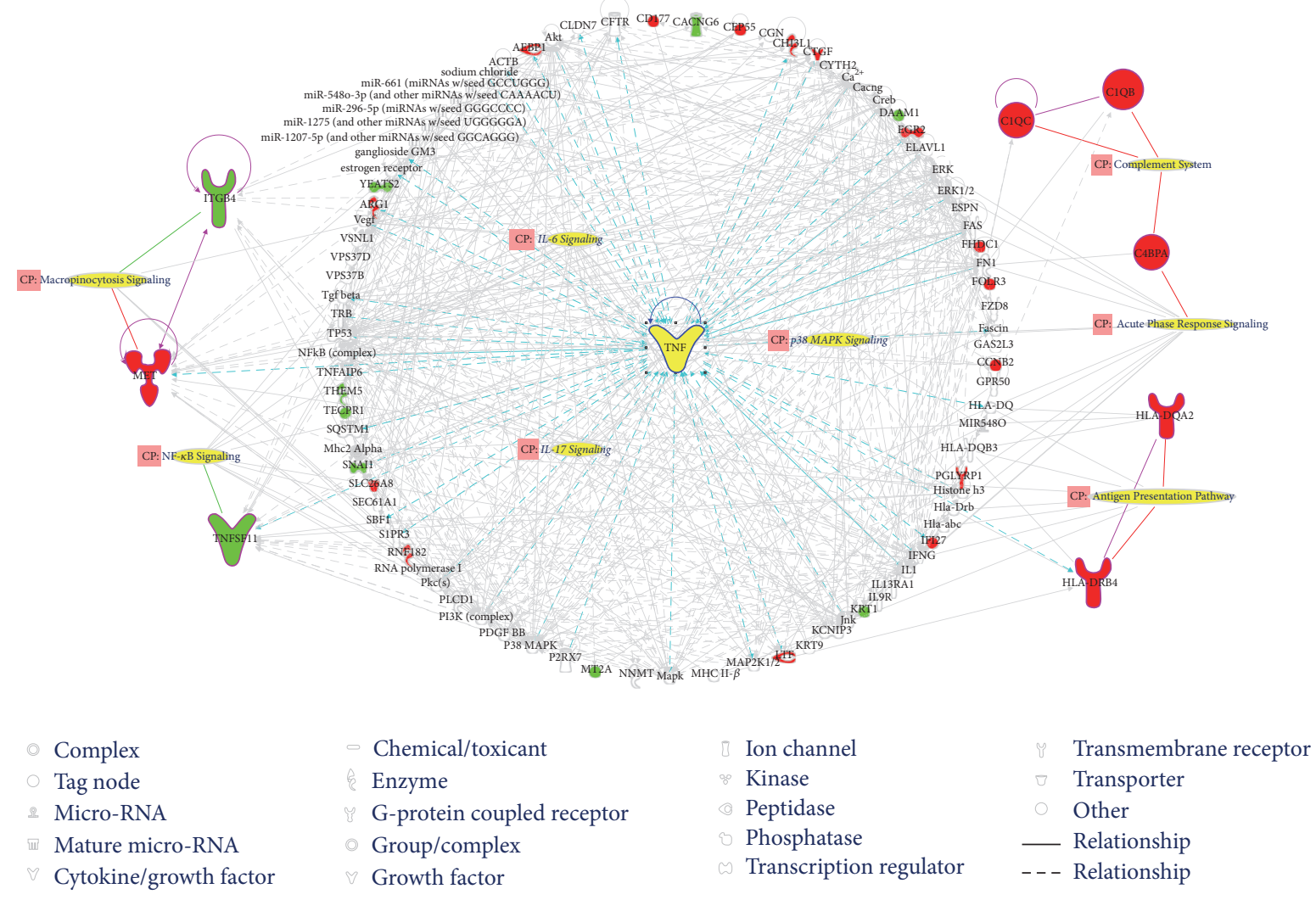

FIGURE 4: The most significantly relevant pathways and highly linked molecules relevant to the merged bionetwork. In the network, molecules are represented as nodes, and the biological relationship between two nodes is represented as a line. Red symbols represent upregulated DEGs; green symbols represent downregulated DEGs. Yellow symbols indicate the highly linked molecules and signaling pathways from the Ingenuity Knowledge Database. "CP" is an abbreviation of "canonical pathway," which represents signaling pathways related to the merged bionetwork. Solid lines between molecules show a direct physical relationship between molecules, whereas dash lines show indirect functional relationships.

HLA-DRB4, and this upregulation is a common mechanism of PS and RA. Therefore, targeting antigen presentation may also be a new strategy for PS treatment. Macropinocytosis represents a distinct pathway of endocytosis in mammalian cells, and it significantly contributes to antigen presentation by the immune system. A study revealed that the type II collagen in an RA mice model was taken up by DCs and macrophages predominantly via inhibition of micropinocytosis [25]. In addition, the nonapoptotic cell death associated with perturbations of micropinocytosis is one apoptosis mechanism caused by RA [26]. This study showed that micropinocytosis signaling is also involved in PS, which offers a new method to understand the pathogenesis of PS. The acute phase response serves as a core of the innate immune response, and proteins relevant to this response were closely correlated with the development of RA [27]. The results from this study suggest that the pathogenesis of both PS and RA might partially result from the perturbation of the acute phase response. Regarding previous studies, the four signaling pathways discussed above are involved in the metabolism and regulation of cytokines. Specifically, when the complement system is stimulated by certain triggers, proteases in the system cleave specific proteins to release cytokines [28]. Antigen presentation plays an important role in cytokine production in PS and RA [29]. Cytokines, such as IFN $\gamma$ and IL-17A, regulate macropinocytosis in macrophages [30]. Cytokine levels (IL-6 and IFN $\gamma$ ) mediate the acute phase response [31]. In short, perturbation of these pathways affected particular cytokines, which potentially partially reflects the pathogenesis of PS and RA.

IL-6 is a proinflammatory cytokine that induces activation of Th cells and controls the balance between Treg cells and Th17 cells. In lesional psoriatic skin, IL-6 is markedly elevated, and T lymphocytes encounter high IL-6 levels, thus allowing cutaneous $\mathrm{T}$ cells to avoid Treg suppression and increasing the Th17 inflammatory activity [32]. Targeting IL-6 signaling in PS may rebalance Treg/Th17 activity and ameliorate the disease [33]. IL-6 also stimulates the inflammatory and autoimmune processes in RA, and both deregulation of IL- 6 production and blockade of IL- 6 signaling are effective strategies in treating experimental models of RA [34]. IL17 is the signature cytokine secreted by Th17 cells. IL-17 is particularly important in PS due to its proinflammatory effects and its involvement in an integrated inflammatory loop with DCs and keratinocytes, contributing to an overproduction of inflammatory cytokines that leads to amplification 


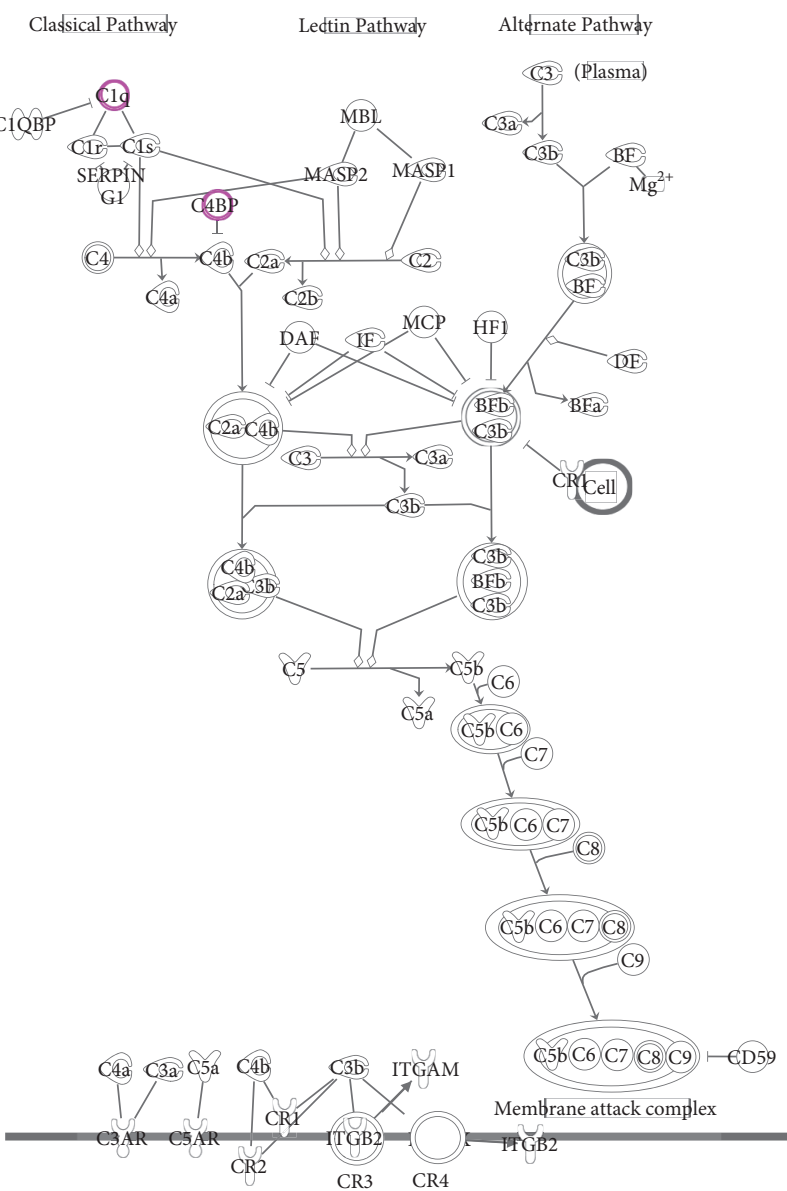

(a)

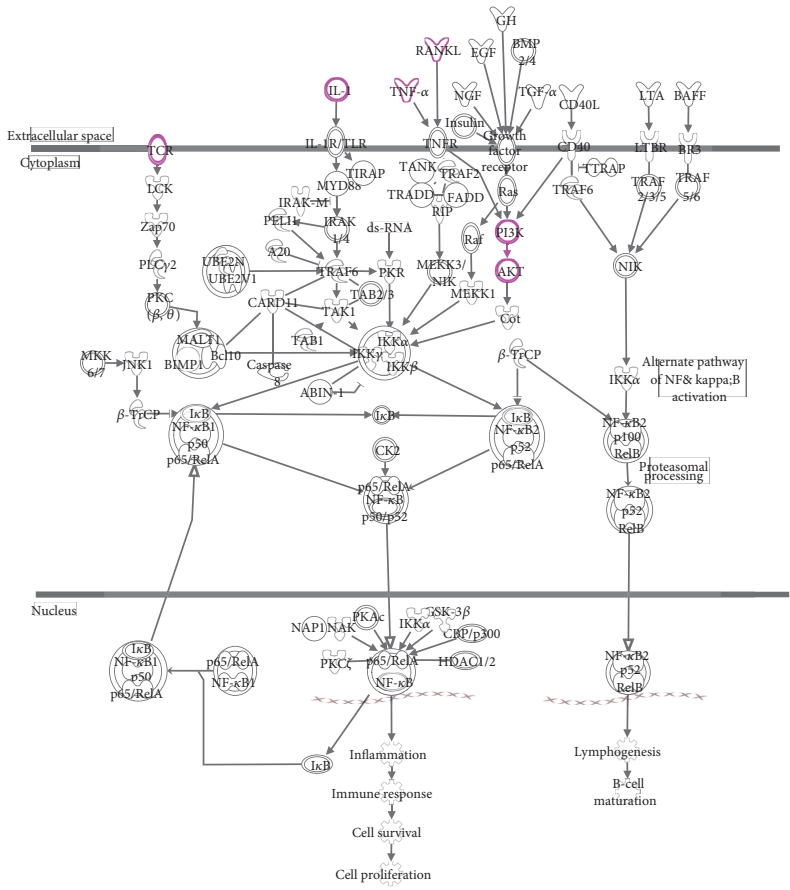

(b)

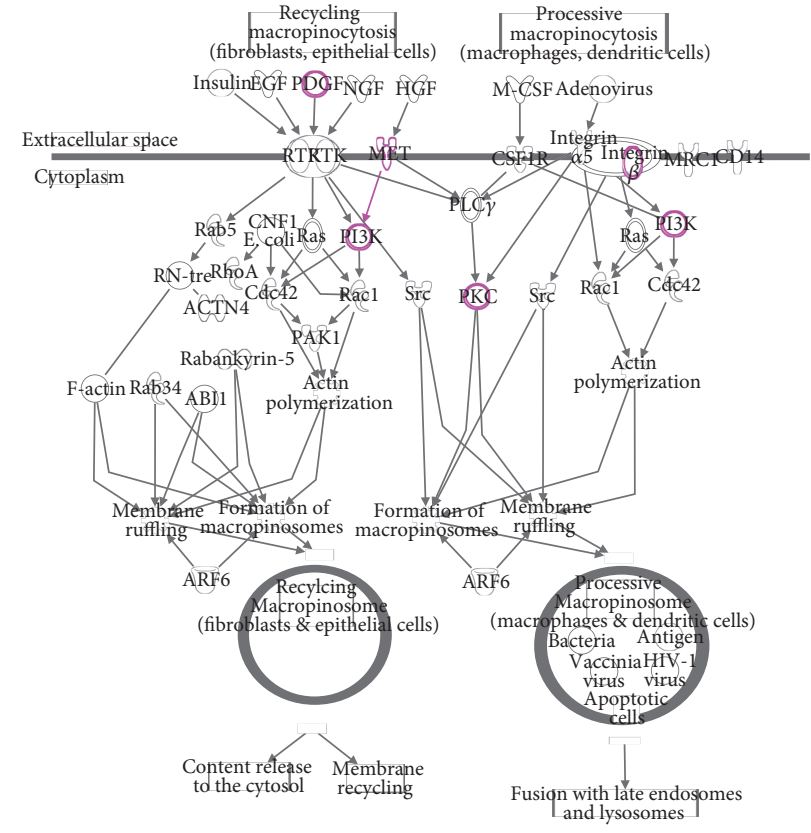

(c)

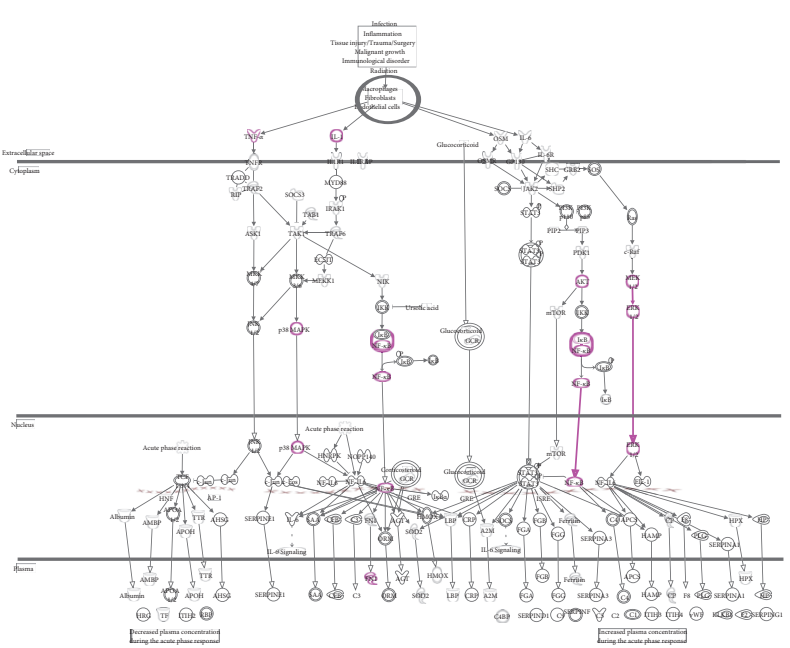

(d)

Figure 5: Continued. 


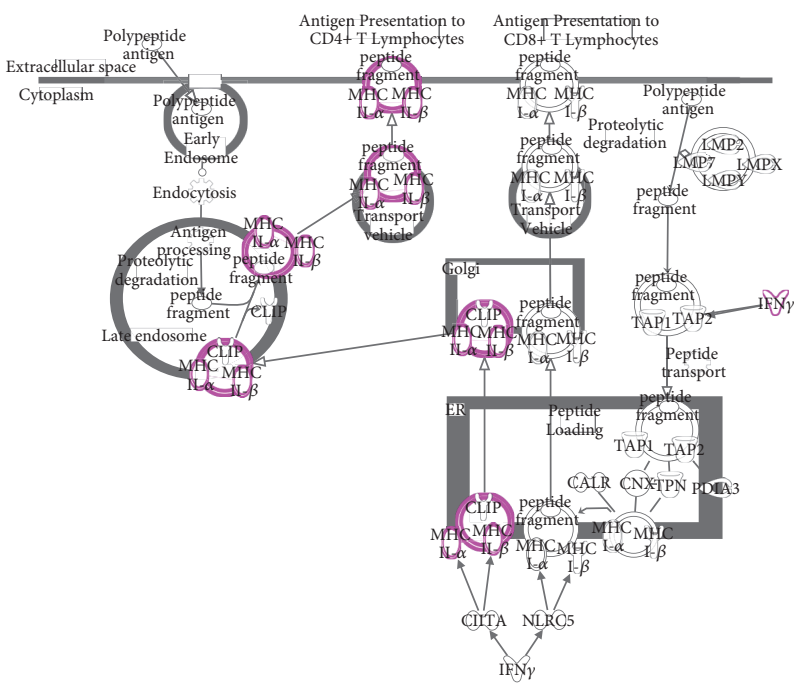

(e)

FIGURE 5: Top five pathways. Purple symbols represent the shared DEGs. (a) Complement system. (b) NF- $\kappa$ B signaling. (c) Macropinocytosis signaling. (d) Acute phase response signaling. (e) Antigen presentation.

of the immune response $[35,36]$. A study demonstrated that effective treatment of PS with TNF inhibitors was associated with suppression of IL-17 signaling [37]. Similarly, the therapeutic strategy of IL-17 signaling inhibition was also used to treat RA [38]. TNF, formerly known as TNF- $\alpha$, is the best-known member of TNF superfamily. As a cytokine, TNF stimulates cell proliferation and cell differentiation and plays a key role in the pathogenesis of PS and RA [39]. A lack of the TNF G allele is associated with reduced PS severity [40]. CSF3 acts as a cytokine and may be produced by the endothelium and by macrophages. A previous study confirmed that CSF3 is a typical IL-17A-regulated gene in the keratinocytes of PS [35]. IFNG encodes the IFN $\gamma$ protein. IFN $\gamma$ is a cytokine secreted by Th cells (specifically, Th1 cells) and is an important activator of macrophages. IFN $\gamma$ has been implicated in the initiation/maintenance of inflammation. A study showed that the pathogenesis of RA was correlated with reduced frequencies of IFN $\gamma$ producers [41]. This study found that these particular cytokines, such as IL-6, IL-17, TNF, CSF3, and IFN $\gamma$, as well as the corresponding signaling pathways are involved in the pathogenesis of PS and RA, which not only verified previous discoveries but also reflected the importance of these cytokines. In brief, an imbalance in these cytokines plays a crucial role in the pathogenesis of PS and RA, and targeting these cytokines is a key strategy for prevention and treatment.

$\mathrm{NF}-\kappa \mathrm{B}$ is essential for the expression of proinflammatory cytokines and controls a number of essential cellular functions, including the immune response, cell proliferation, and apoptosis. Loss of normal NF- $\kappa \mathrm{B}$ signaling regulation is a major contributor to a variety of inflammatory and autoimmune diseases, such as PS and RA [42, 43]. Activator protein-1 (AP-1) is recognized as a regulator of the expression of cytokines, such as CSF3, IL-6, and TNF, and is causally involved in PS and RA [44]. The immediate early gene product Fos is part of the AP-1 transcription factor, and its deregulation is associated with a variety of immunological defects. Selective inhibition of Fos function demonstrated that targeting Fos/AP-1 activity could be an promising new option for arthritis treatment [45]. ERK1/2 and p38 MAPK are important members of the MAPK family, which are responsive to inflammatory cytokines, and they are involved in cell differentiation, apoptosis, and autophagy. ERK1/2 is phosphorylated and activated via cell surface receptors stimulated by cytokines. Emerging data suggest that cytokine expression in response to p38 MAPK and ERK1/2 activation is involved in the etiopathogenesis of PS and that p38 MAPK signaling is an indicator of the loss of keratinocyte cellcell adhesion in PS $[46,47]$. IL- 6, NF- $\kappa$ B, and p38 MAPK signaling activation is an important characteristic of the inflammatory response in activated macrophages in RA, and p38 MAPK signaling is involved in the process of RA angiogenesis [48]. In addition, suppressing the expression of TNF- $\alpha$ and IL- 6 through inhibiting the activation of NF- $\kappa \mathrm{B}$ and ERK1/2 is an important strategy for treating RA [49]. This study indicated that four biomolecules, NF- $\kappa$ B, Fos, p38 MAPK, and ERK1/2, are highly linked molecules or upstream regulators that are closely associated with the shared DEGs in PS and RA and therefore regulated cytokines; these four biomolecules represent novel targets to prevent and treat these two diseases.

\section{Conclusions}

The common pathogenesis of PS and RA was characterized by a cytokine imbalance. The deregulation of certain key molecules, such as ERK1/2, CSF3, FOS, IFNG, and TNF, as well as the perturbation of signaling pathways, including the complement system, antigen presentation, macropinocytosis signaling, NF- $\kappa \mathrm{B}$ signaling, IL-6 signaling, IL-17 signaling, 

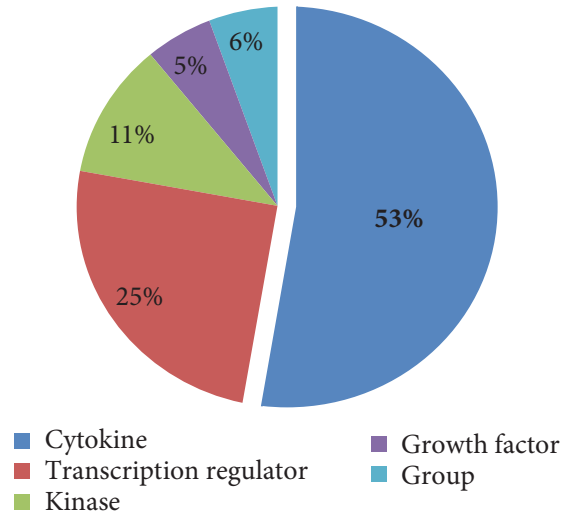

(a)

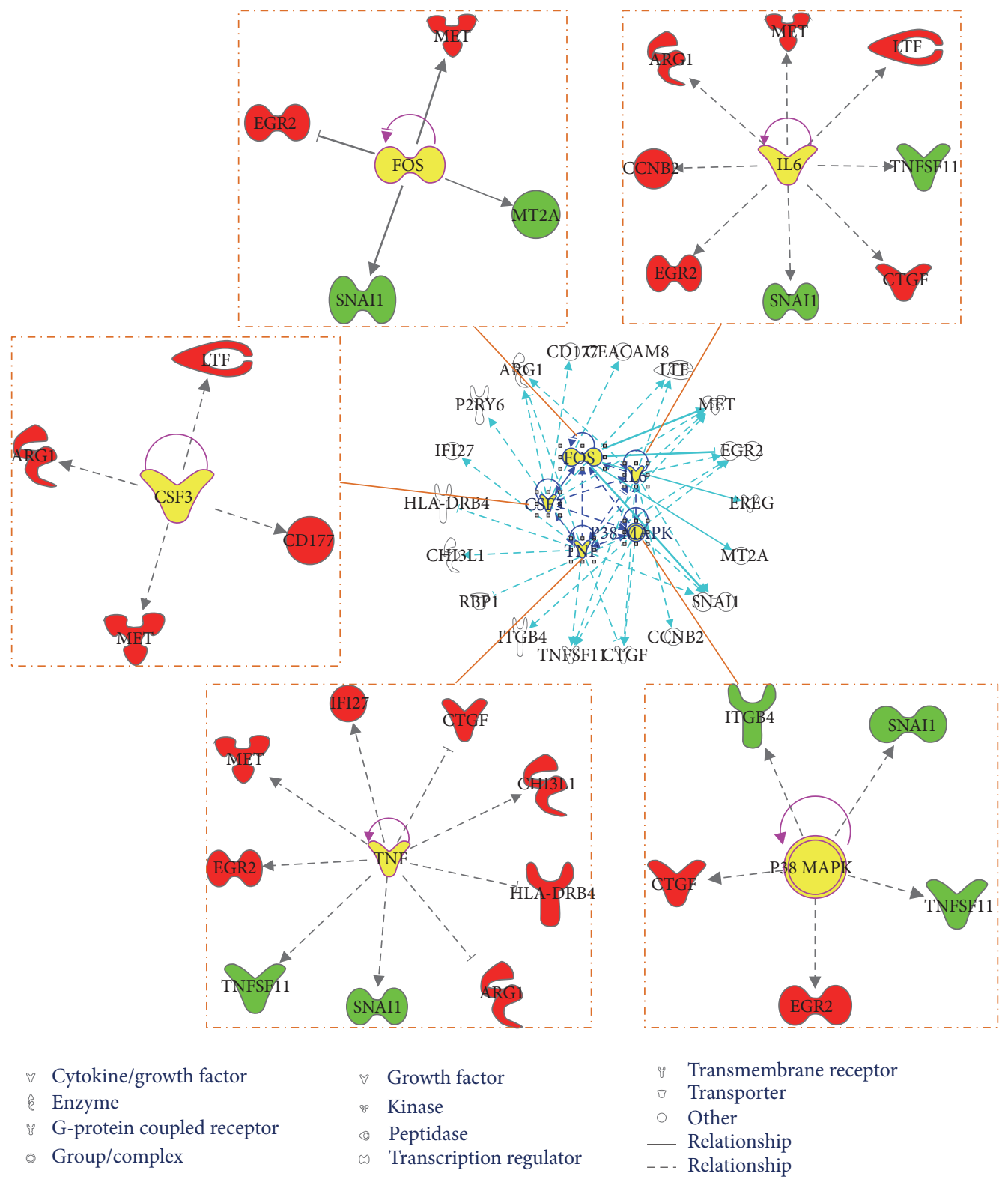

(b)

Figure 6: Upstream regulators relevant to the shared to DEGs. (a) The percentage of molecule type of upstream regulator. (b) Top five upstream regulators. Red nodes represent upregulated DEGs; green nodes represent downregulated DEGs. Yellow nodes represent the upstream regulators. Solid lines between molecules indicate a direct physical relationship between molecules, whereas dash lines indicate indirect functional relationships. 


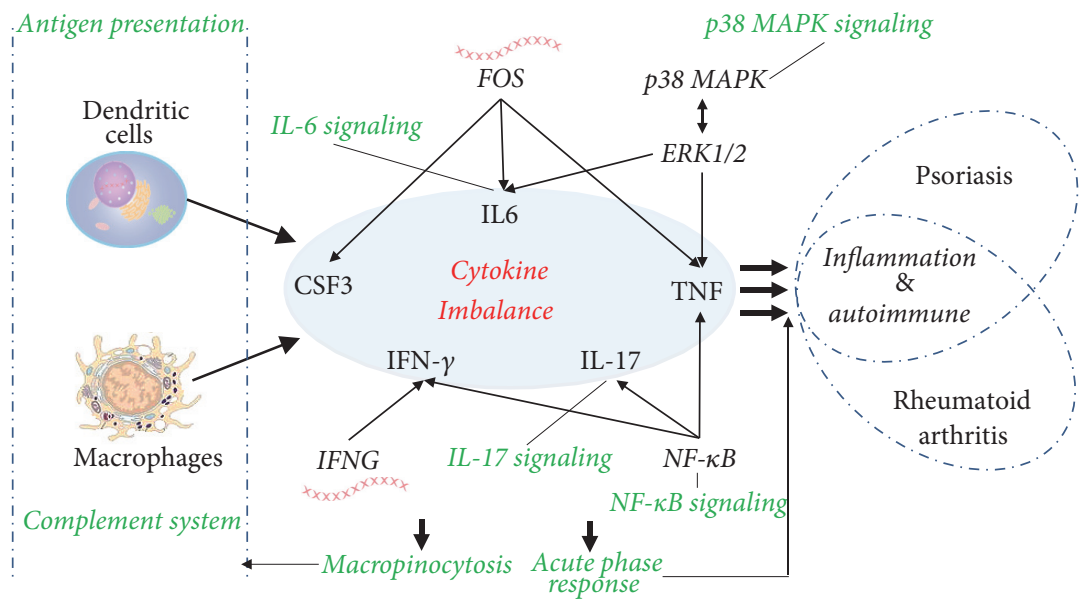

FIGURE 7: The common molecular mechanisms of PS and RA are characterized by a cytokine imbalance. Arrows represent the relationship between molecules or between molecules and cells. Green terms represent signaling pathways. The molecules in ovals are cytokines.

and p38 MAPK signaling, reflected this type of imbalance. The new findings in this study provided a new molecular understanding of PS and RA and could pave the road for the discovery of new strategies for treating PS and RA.

\begin{tabular}{ll} 
Abbreviations \\
PS: & Psoriasis \\
RA: & Rheumatoid arthritis \\
PBMCs: & Peripheral blood mononuclear cells \\
PV: & Psoriasis vulgaris \\
PASI: & Psoriasis area severe index \\
BSA: & Body surface area \\
DAS28: & Disease activity score in 28 joints \\
BMI: & Body mass index \\
ESR: & Erythrocyte sedimentation rate \\
CRP: & C-reactive protein \\
RF: & Rheumatoid factor \\
WBC: & White blood cell \\
HGB: & Hemoglobin \\
PLT: & Platelets \\
IL: & Interleukin \\
Th: & Thelper \\
Treg: & Regulatory T cells \\
TNF: & Tumor necrosis factor \\
CSF3: & Colony-stimulating factor 3 \\
IFN $\gamma:$ & Interferon gamma \\
IFNG: & Interferon gene \\
NF- $\kappa$ B: & Nuclear factor-kappa B \\
MAPKs: & Mitogen-activated protein kinases \\
ERK1/2: & Extracellular signal-regulated kinase 1/2 \\
NGS: & Next-generation sequencing. \\
& \\
\hline &
\end{tabular}

\section{Competing Interests}

All of the authors declare that there are no competing interests regarding the publication of this paper.

\section{Authors' Contributions}

Cheng Xiao, Aiping Lu, and Youwen Zhou conceived and designed the experiments. Cheng Lu, Xuyan Niu, Yanping Bai, and Cheng Xiao performed the experiments. Yong Tan, Xuyan Niu, Chunyan Jiang, and Yang Wang analyzed the data. Aiping $\mathrm{Lu}$ and Cheng Xiao contributed reagents/materials/analysis tools. Yong Tan, Qiu Qi, and Cheng Lu wrote the paper. Yong Tan, Qiu Qi, and Cheng Lu contributed equally to this work.

\section{Acknowledgments}

This study was supported by the International Cooperation Project of the Ministry of Science and Technology (no. 2014DFA31490), the National Science Foundation of China (no. 81373773), the Science and Technology Projects for Supervisors of Beijing Outstanding Doctorate Dissertation (no. 20118450201), the Beijing Nova Program (no. xx2014B073), and the Beijing Municipal Administration of Hospitals "Youth Programme" (no. QML20150603).

\section{References}

[1] N. N. Mehta, Y. Yu, R. Pinnelas et al., "Attributable risk estimate of severe psoriasis on major cardiovascular events," American Journal of Medicine, vol. 124, no. 8, pp. 775.e1-775.e6, 2011.

[2] D. H. Solomon, N. J. Goodson, J. N. Katz et al., "Patterns of cardiovascular risk in rheumatoid arthritis," Annals of the Rheumatic Diseases, vol. 65, no. 12, pp. 1608-1612, 2006.

[3] A. B. Kimball, J. Schenfeld, N. A. Accortt, M. S. Anthony, K. J. Rothman, and D. Pariser, "Incidence rates of malignancies and hospitalized infectious events in patients with psoriasis with or without treatment and a general population in the U.S.A.: 200509," British Journal of Dermatology, vol. 170, no. 2, pp. 366-373, 2014.

[4] L. Mellemkjær, M. S. Linet, G. Gridley, M. Frisch, H. Møller, and J. H. Olsen, "Rheumatoid arthritis and cancer risk," European Journal of Cancer, vol. 32, no. 10, pp. 1753-1757, 1996. 
[5] G. Ganzetti, A. Campanati, E. Molinelli, and A. Offidani, "Psoriasis, non-alcoholic fatty liver disease, and cardiovascular disease: three different diseases on a unique background," World Journal of Cardiology, vol. 8, no. 2, pp. 120-131, 2016.

[6] E. M. Ruderiman, J. M. Crawford, A. Maier, J. J. Liu, E. M. Gravallese, and M. E. Weinblatt, "Histologic liver abnormalities in an autopsy series of patients with rheumatoid arthritis," British Journal of Rheumatology, vol. 36, no. 2, pp. 210-213, 1997.

[7] S. Kagami, H. L. Rizzo, J. J. Lee, Y. Koguchi, and A. Blauvelt, "Circulating Th17, Th22, and Th1 cells are increased in psoriasis," Journal of Investigative Dermatology, vol. 130, no. 5, pp. 13731383, 2010.

[8] A. Oka, T. Mabuchi, A. Ozawa, and H. Inoko, "Current understanding of human genetics and genetic analysis of psoriasis," Journal of Dermatology, vol. 39, no. 3, pp. 231-241, 2012.

[9] A. Michalak-Stoma, A. Pietrzak, J. C. Szepietowski, A. Zalewska-Janowska, T. Paszkowski, and G. Chodorowska, "Cytokine network in psoriasis revisited," European Cytokine Network, vol. 22, no. 4, pp. 160-168, 2011.

[10] E. Choy, "Understanding the dynamics: pathways involved in the pathogenesis of rheumatoid arthritis," Rheumatology (Oxford), vol. 51, supplement 5, pp. v3-v11, 2012.

[11] E. R. Mardis, "The impact of next-generation sequencing technology on genetics," Trends in Genetics, vol. 24, no. 3, pp. 133-141, 2008.

[12] V. H. Teixeira, R. Olaso, M.-L. Martin-Magniette et al., "Transcriptome analysis describing new immunity and defense genes in peripheral blood mononuclear cells of rheumatoid arthritis patients," PLOS ONE, vol. 4, Article ID e6803, 2009.

[13] B. Li, L. C. Tsoi, W. R. Swindell et al., "Transcriptome analysis of psoriasis in a large case-control sample: RNA-seq provides insights into disease mechanisms," Journal of Investigative Dermatology, vol. 134, no. 7, pp. 1828-1838, 2014.

[14] A. Menter, A. Gottlieb, S. R. Feldman et al., "Guidelines of care for the management of psoriasis and psoriatic arthritis: section 1. Overview of psoriasis and guidelines of care for the treatment of psoriasis with biologics," Journal of the American Academy of Dermatology, vol. 58, no. 5, pp. 826-850, 2008.

[15] Psoriasis Study Group svdboCMA, "Guideline for the treatment of psoriasis (2008)," Chinese Journal of Dermatology, vol. 3, pp. 213-214, 2008.

[16] A. J. Silman and J. E. Pearson, "Epidemiology and genetics of rheumatoid arthritis," Arthritis Research \& Therapy, vol. 4, supplement 3, pp. S265-S272, 2002.

[17] X. Niu, C. Lu, C. Xiao et al., "The shared crosstalk of multiple pathways involved in the inflammation between rheumatoid arthritis and coronary artery disease based on a digital gene expression profile," PLoS ONE, vol. 9, no. 12, Article ID el13659, 2014.

[18] A. S. Morrissy, R. D. Morin, A. Delaney et al., "Next-generation tag sequencing for cancer gene expression profiling," Genome Research, vol. 19, no. 10, pp. 1825-1835, 2009.

[19] W. R. Swindell, M. K. Sarkar, Y. Liang, X. Xing, and J. E. Gudjonsson, "Cross-disease transcriptomics: unique IL-17A signaling in psoriasis lesions and an autoimmune PBMC signature," Journal of Investigative Dermatology, vol. 136, no. 9, pp. 18201830, 2016.

[20] X. Niu, C. Lu, C. Xiao et al., "The crosstalk of pathways involved in immune response maybe the shared molecular basis of rheumatoid arthritis and type 2 diabetes," PLoS ONE, vol. 10, no. 8, Article ID e0134990, 2015.
[21] N. S. Merle, R. Noe, L. Halbwachs-Mecarelli, V. FremeauxBacchi, and L. T. Roumenina, "Complement system part II: role in immunity," Frontiers in Immunology, vol. 6, article 257, 2015.

[22] E. Ballanti, C. Perricone, G. Di Muzio et al., "Role of the complement system in rheumatoid arthritis and psoriatic arthritis: relationship with anti-TNF inhibitors," Autoimmunity Reviews, vol. 10, no. 10, pp. 617-623, 2011.

[23] J. L. Rodríguez-Fernández, "Antigen presentation by dendritic cells in rheumatoid arthritis," Current Topics in Medicinal Chemistry, vol. 13, no. 6, pp. 712-719, 2013.

[24] E. G. Spack, "Treatment of autoimmune diseases through manipulation of antigen presentation," Critical Reviews in Immunology, vol. 17, no. 5-6, pp. 529-536, 1997.

[25] A. von Delwig, C. M. U. Hilkens, D. M. Altmann et al., "Inhibition of macropinocytosis blocks antigen presentation of type II collagen in vitro and in vivo in HLA-DR1 transgenic mice," Arthritis Research and Therapy, vol. 8, no. 4, article no. R93, 2006.

[26] W. A. Maltese and J. H. Overmeyer, "Non-apoptotic cell death associated with perturbations of macropinocytosis," Frontiers in Physiology, vol. 6, article no. 38, 2015.

[27] C. Cray, J. Zaias, and N. H. Altman, "Acute phase response in animals: a review," Comparative Medicine, vol. 59, no. 6, pp. 517526, 2009.

[28] N. S. Merle, S. E. Church, V. Fremeaux-Bacchi, and L. T. Roumenina, "Complement system part I-molecular mechanisms of activation and regulation," Frontiers in Immunology, vol. 6, article no. 262, 2015.

[29] M. Feldmann, "Molecular mechanisms involved in human autoimmune diseases: relevance of chronic antigen presentation. Class II expression and cytokine production," Immunology, vol. 2, pp. 66-71, 1989.

[30] D. R. Michael, T. G. Ashlin, C. S. Davies et al., "Differential regulation of macropinocytosis in macrophages by cytokines: implications for foam cell formation and atherosclerosis," Cytokine, vol. 64, no. 1, pp. 357-361, 2013.

[31] F. Martín, F. Santolaria, N. Batista et al., "Cytokine levels (IL6 and IFN- $\gamma$ ), acute phase response and nutritional status as prognostic factors in lung cancer," Cytokine, vol. 11, no. 1, pp. 80-86, 1999.

[32] W. A. Goodman, A. D. Levine, J. V. Massari, H. Sugiyama, T. S. McCormick, and K. D. Cooper, "IL-6 signaling in psoriasis prevents immune suppression by regulatory T cells," Journal of Immunology, vol. 183, no. 5, pp. 3170-3176, 2009.

[33] P. G. Baraldi, B. Cacciari, A. Guiotto, R. Romagnoli, G. Spalluto, and A. N. Zaid, "Synthesis, cytotoxicity and antitumor activity of some new simplified pyrazole analogs of the antitumor agent CC-1065. Effect of an hydrophobic group on antitumor activity," Farmaco, vol. 52, no. 12, pp. 711-716, 1997.

[34] P. Ataie-Kachoie, M. H. Pourgholami, and D. L. Morris, "Inhibition of the IL- 6 signaling pathway: a strategy to combat chronic inflammatory diseases and cancer," Cytokine and Growth Factor Reviews, vol. 24, no. 2, pp. 163-173, 2013.

[35] R. Muromoto, T. Hirao, K. Tawa et al., "IL-17A plays a central role in the expression of psoriasis signature genes through the induction of $\mathrm{I} \kappa \mathrm{B}-\zeta$ in keratinocytes," International Immunology, vol. 28, no. 9, pp. 443-452, 2016.

[36] S. Klebow, M. Hahn, A. Nikoalev et al., "IL-6 signaling in myelomonocytic cells is not crucial for the development of IMQ-induced psoriasis," PLoS ONE, vol. 11, no. 3, Article ID e0151913, 2016. 
[37] L. C. Zaba, M. Suárez-Fariñas, J. Fuentes-Duculan et al., "Effective treatment of psoriasis with etanercept is linked to suppression of IL-17 signaling, not immediate response TNF genes," Journal of Allergy and Clinical Immunology, vol. 124, no. 5, pp. 1022-1030.e395, 2009.

[38] Q. Luo, Y. Sun, W. Liu et al., "A novel disease-modifying antirheumatic drug, iguratimod, ameliorates murine arthritis by blocking IL-17 signaling, distinct from methotrexate and leflunomide," Journal of Immunology, vol. 191, no. 10, pp. 49694978, 2013.

[39] B. Zhao, S. N. Grimes, S. Li, X. Hu, and L. B. Ivashkiv, “TNFinduced osteoclastogenesis and inflammatory bone resorption are inhibited by transcription factor RBP-J," Journal of Experimental Medicine, vol. 209, no. 2, pp. 319-334, 2012.

[40] S. Popadic, E. Savic, M. Markovic et al., "TNF, IL12B, and IFNG gene polymorphisms in serbian patients with psoriasis," Annals of Dermatology, vol. 27, no. 2, pp. 128-132, 2015.

[41] J. Dong, H.-D. Chang, C. Ivascu et al., "Loss of methylation at the IFNG promoter and CNS-1 is associated with the development of functional IFN- $\gamma$ memory in human CD4+ T lymphocytes," European Journal of Immunology, vol. 43, no. 3, pp. 793-804, 2013.

[42] R. Li, J. Wang, X. Wang et al., "Increased $\beta \operatorname{TrCP}$ are associated with imiquimod-induced psoriasis-like skin inflammation in mice via NF- $\kappa$ B signaling pathway," Gene, vol. 592, no. 1, pp. 164-171, 2016.

[43] L. A. Criswell, "Gene discovery in rheumatoid arthritis highlights the CD40/NF- $\kappa$ B signaling pathway in disease pathogenesis," Immunological Reviews, vol. 233, no. 1, pp. 55-61, 2010.

[44] R. Zenz, R. Eferl, C. Scheinecker et al., "Activator protein 1 (Fos/Jun) functions in inflammatory bone and skin disease," Arthritis Research and Therapy, vol. 10, no. 1, article 201, 2008.

[45] M. Durchdewald, P. Angel, and J. Hess, "The transcription factor Fos: a Janus-type regulator in health and disease," Histology and Histopathology, vol. 24, no. 11, pp. 1451-1461, 2009.

[46] A. Mavropoulos, E. I. Rigopoulou, C. Liaskos, D. P. Bogdanos, and L. I. Sakkas, "The role of p38 MAPK in the aetiopathogenesis of psoriasis and psoriatic arthritis," Clinical and Developmental Immunology, vol. 2013, Article ID 569751, 8 pages, 2013.

[47] C. Johansen, K. Kragballe, M. Westergaard, J. Henningsen, K. Kristiansen, and L. Iversen, "The mitogen-activated protein kinases p38 and ERK1/2 are increased in lesional psoriatic skin," British Journal of Dermatology, vol. 152, no. 1, pp. 37-42, 2005.

[48] C. Hong, C. Shen, H. Ding et al., "An involvement of SRB1 mediated p38 MAPK signaling pathway in serum amyloid A-induced angiogenesis in rheumatoid arthritis," Molecular Immunology, vol. 66, no. 2, pp. 340-345, 2015.

[49] M. Tsubaki, T. Takeda, T. Kino et al., "Mangiferin suppresses CIA by suppressing the expression of TNF- $\alpha$, IL-6, IL- $1 \beta$, and RANKL through inhibiting the activation of NF- $\kappa \mathrm{B}$ and ERK1/2," American Journal of Translational Research, vol. 7, no. 8, pp. 1371-1381, 2015. 


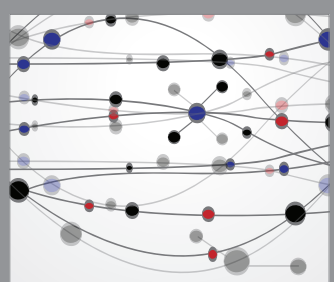

The Scientific World Journal
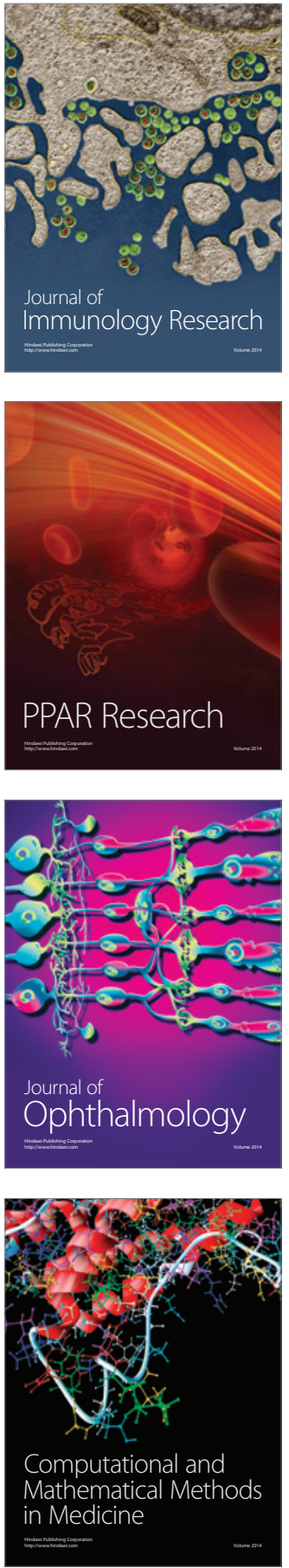

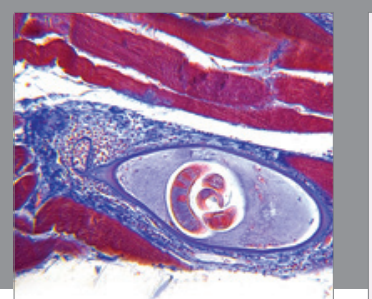

Gastroenterology Research and Practice
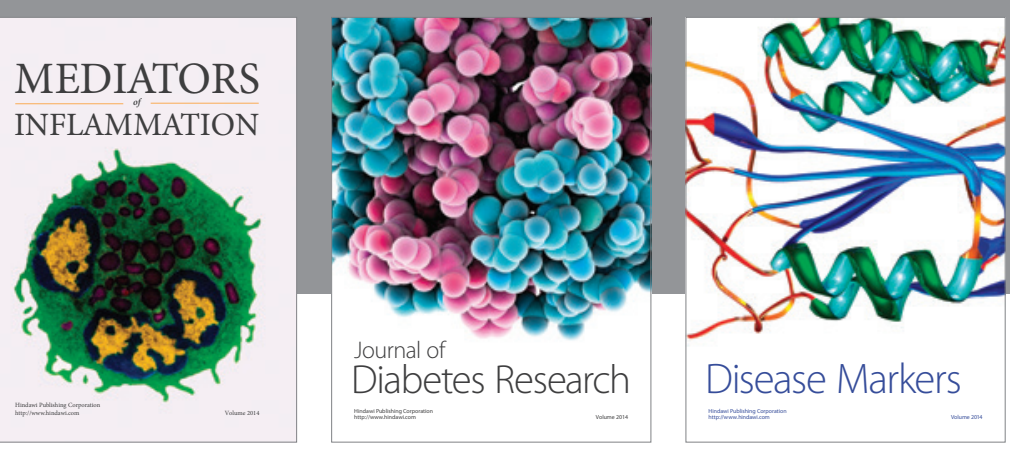

Disease Markers

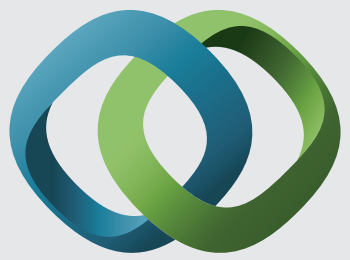

\section{Hindawi}

Submit your manuscripts at

https://www.hindawi.com
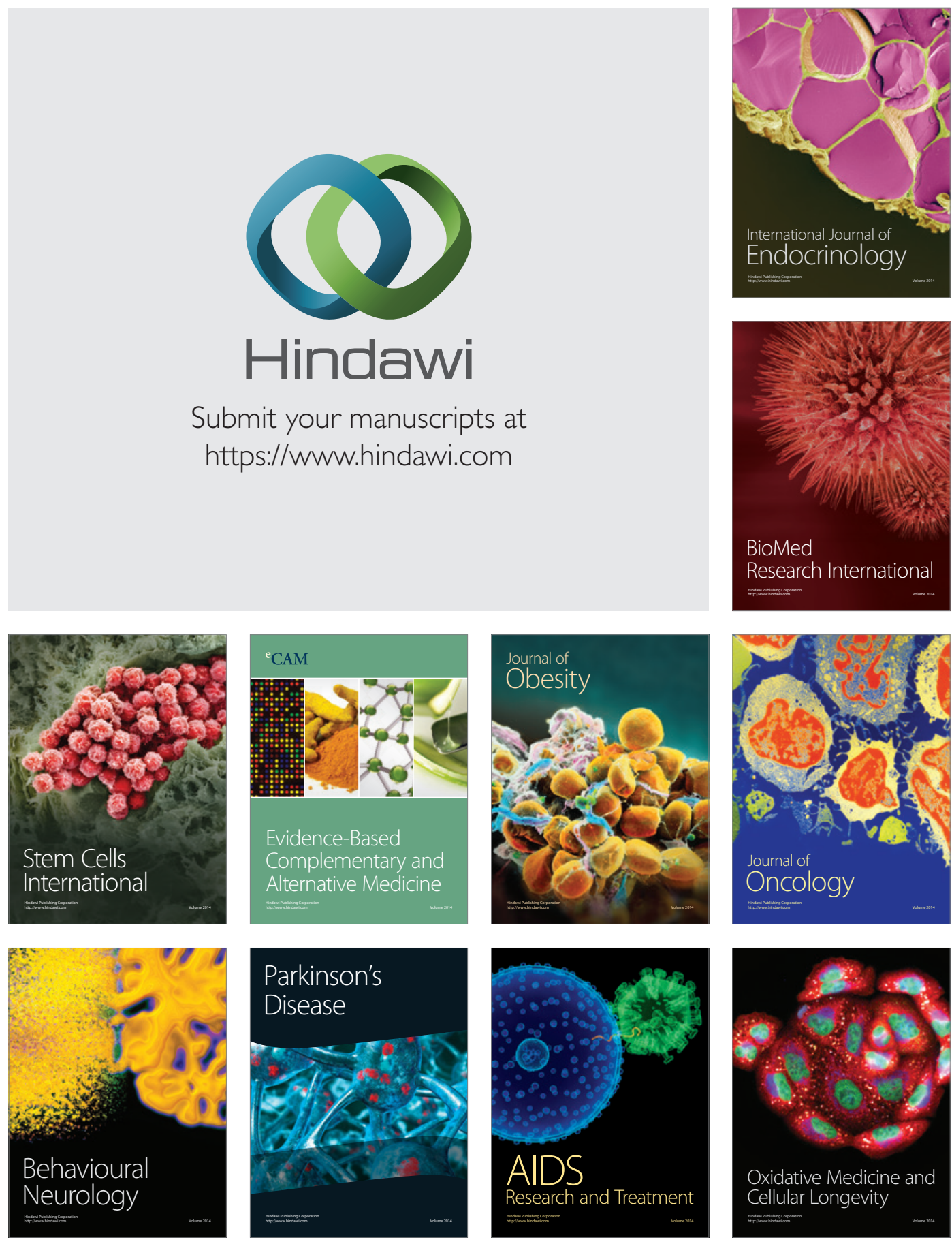